\title{
Interpretation of Chlamydia trachomatis antibody response in chlamydial oculogenital infection
}

\author{
H C Patel, B T Goh, N D Viswalingam, J D Treharne
}

\begin{abstract}
Objective-To study: (a) the chlamydial antibody response (to the $D-K$ serovars) using the micro-immunofluorescence (micro-IF) test in the following groups: (I) chlamydial genital infection only, (II) chlamydial ocular infection only, (III) combined chlamydial ocular and genital infection (oculo-genital infection), (IV) chlamydial ocular infection with chlamydia-negative non-gonococcal urethritis, (V) adenovirus conjunctivitis (control group 1), (VI) male partners of group I-IV with no chlamydial oculogenital infection or non-gonococcal urethritis (control group 2) (b) the cross reactivity of antibodies in patients' sera between the three chlamydial species and within the serovars of $C$ trachomatis in those with culture-positive chlamydial oculogenital infection.

Setting-oculogenital (diagnostic) clinic
\end{abstract} at Moorfields Eye Hospital, London, UK.

Subjects-209 consecutive patients attending the clinic with Chlamydia trachomatis oculogenital infection and 86 patients with adenovirus conjunctivitis (control group 1) and 55 male partners with no evidence of chlamydial oculogenital infection or non-gonococcal urethritis (control group 2).

Results-Of all the patients with proven chlamydial oculogenital infection, $10 \cdot 5 \%$ (22/209) and 94\% (197/209) had IgM and IgG antibodies respectively. The geometric mean IgG antibody titres (GMT) were $1: 98,1: 123,1: 245$ and $1: 101$ in groups $I$ to IV respectively. The IgG GMT values seen in control groups 1 and 2 were $1: 45$ and 1:36 respectively. Only $2 / 86(2 \%)$ patients in group $V$ (control group 1) had IgG chlamydial antibodies of $1: 32$ and $1: 64$, whilst only $1 / 55(1 \cdot 8 \%)$ and $4 / 55(7 \cdot 3 \%)$ of patients in group VI(control group 2) had chlamydial IgG antibody titres of $\geqslant 1: 256$ and $\geqslant 1: 128$ respectively. $A$ four-fold rise or fall in IgG antibody titre occurred in $56 \%(107 / 192)$ of patient groups I-IV over 2-6 weeks. Low titre cross-reactive antibody responses against different chlamydial species and serovars were commonly seen; $71 \%(148 / 209)$ of all patients showed cross-reactivity with Chlamydia pneumoniae or psittaci species or both, whilst $92 \%(193 / 209)$ of patients showed some level of crossreactivity to other pooled serovars of $C$ trachomatis (A-C and L 1-3).
Conclusions-Serological diagnosis of chlamydial infection as evidenced by a positive IgM antibody response, high IgG titre $(\geqslant 1: 256)$ or $\geqslant 4$-fold rise or fall in IgG antibody titre was seen in $78 \%(163 / 209)$ of patients with culture-positive chlamydial oculogenital infection. Chlamydial IgG antibody titres of $\geqslant 1: 256$ had a sensitivity of $42 \cdot 6 \%$, specificity of $98 \cdot 2 \%$, positive predictive value of $98.8 \%$ and a negative predictive value of $31 \%$ for chlamydial infection at any site, when considering groups I-IV and control group 2. In this study of 216 patients with conjunctivitis, a positive IgG antibody response (titre $\geqslant 1: 16$ ) had a sensitivity of $98 \cdot 5 \%$, specificity of $97.7 \%$, positive predictive value of $98.5 \%$ and a negative predictive value of $97.7 \%$, for chlamydial conjunctivitis. Patients with dual chlamydial infection of conjunctiva and genital tract had a higher IgG GMT titre than those with ocular or genital infection alone: infection at a second site may produce an anamnestic response. Although the micro-IF test is a useful adjunct for the diagnosis of chlamydial infection, cross-reactivity between different chlamydial species and serovars is common. Chlamydial seroepidemiological studies should be interpreted with caution, as studies may attribute a serological response to a particular species or serovar in a setting where two or more are prevalent.

\section{(Genitourin Med 1995;71:94-97)}

Keywords: Chlamydia trachomatis; antibody; oculogenital

\section{Introduction}

Three major problems have precluded the successful use of serological tests for the diagnosis of an individual patient's chlamydial genital infection. First, the baseline prevalence of antibody in populations which are sexually active and likely to be at risk for Chlamydia trachomatis infection is high, often ranging from 45 to $65 \%$ of persons tested. ${ }^{1}$ Also little is known about the predictive value of a single antibody test.

The second major difficulty precluding effective use of serodiagnosis results from the asymptomatic or minimally symptomatic nature of many chlamydial infections. Lack of an abrupt onset of symptoms means that many patients are seen during periods when IgM antibody or rising or falling titres of $\operatorname{IgG}$ antibody cannot be demonstrated. 
The third major problem is the cross-reactivity between the different species of chlamydia as well as between different serovars of $C$ trachomatis. This makes it difficult to interpret an antibody response to a particular species in a setting where two or more chlamydial species may be prevalent, for example $C$ trachomatis and $C$ pneumoniae, and also this also questions the validity of studies attributing various clinical syndromes to $C$ trachomatis or other species based on serological evidence alone.

Early serological tests for the diagnosis of chlamydial infection included the complement fixation test (CFT) which may be useful for the diagnosis of the systemic chlamydial infections, lympho-granuloma venereum and psittacosis. However the CFT only detects antibody to the heat-stable, lipopolysaccharide antigen common to all chlamydia species and cannot distinguish between infections due to $C$ trachomatis, $C$ psittaci or $C$ pneumoniae. ${ }^{2}$ In 1970, Wang and Grayston described an indirect immunofluorescence serotyping test (micro-IF), which has led to the elucidation of 15 different serovars of $C$ trachomatis, and has now become accepted as the serological standard. The detection of type-specific antibodies using all 15 serovar antigens can be time-consuming and expensive and modifications of the original test have been described using pooled serologically related antigens, ${ }^{3}$ or pooled, epidemiologically related serovars. ${ }^{4}$ In sera from patients with chlamydial infections, broadly cross-reactive antibody responses to different species and serovars of chlamydia may commonly occur. We were interested to study the micro-IF chlamydial antibody response to the $\mathrm{D}-\mathrm{K}$ and cross-reactivity between the A-C, D-K and L 1-3 serovars as well as between the different species of chlamydia, seen in patients with culture proven chlamydial oculogenital infection. In particular, we wanted to ascertain the role of the serological test in diagnosing chlamydial oculo-genital infection.

Table 1 IgM and IgG response in chlamydial oculo-genital infection

\begin{tabular}{|c|c|c|c|c|c|}
\hline \multirow{2}{*}{$\begin{array}{l}\text { Infection } \\
\text { Site }\end{array}$} & \multirow[b]{2}{*}{ Total } & \multicolumn{2}{|l|}{$I g M$} & \multicolumn{2}{|l|}{$I g G$} \\
\hline & & - & + & - & + \\
\hline $\begin{array}{l}\text { Genital } \\
\text { Ocular } \\
\text { Oculogenital } \\
\text { Ocular \& NSU }\end{array}$ & $\begin{array}{l}79(100 \%) \\
20(100 \%) \\
80(100 \%) \\
30(100 \%)\end{array}$ & $\begin{array}{l}70(87 \%)^{\star} \\
16(75 \%) \\
73(90 \%) \dagger \\
28(93 \%) \ddagger\end{array}$ & $\begin{array}{l}9(13 \%) \\
4(25 \%) \\
7(10 \%) \\
2(7 \%)\end{array}$ & $\begin{array}{l}10(13 \%) \\
1 \\
0 \\
1\end{array}$ & $\begin{array}{l}69(87 \%) \\
19(95 \%) \\
80(100 \%) \\
29(97 \%)\end{array}$ \\
\hline Total & $209(100 \%)$ & $187(89 \cdot 5 \%)$ & $22(10 \cdot 5 \%)$ & $12(6 \%)$ & $197(94 \%)$ \\
\hline
\end{tabular}

Titre $\geqslant=1: 16$

$\star_{2}$ had subsequent IgM ab

t4 had subsequent IgM ab

$\ddagger 1$ had subsequent IgM ab

Table 2 Chlamydia serial IgG antibody response in oculo-genital infection

\begin{tabular}{lcrrr}
\hline Site of Infection & No Change & $\geqslant 4$ fold $\uparrow$ & $\geqslant 4$ fold $\downarrow$ & \multicolumn{1}{c}{ Total } \\
\hline Ocular & $6(35 \%)$ & $8(47 \%)$ & $3(18 \%)$ & $17(100 \%)$ \\
Genital & $40(55 \%)$ & $13(18 \%)$ & $20(27 \%)$ & $73(100 \%)$ \\
Oculo-Genital & $30(41 \%)$ & $7(9 \%)$ & $37(50 \%)$ & $74(100 \%)$ \\
Ocular \& NSU & $9(32 \%)$ & $10(36 \%)$ & $9(32 \%)$ & $28(100 \%)$ \\
Total & $85(44 \%)$ & $38(20 \%)$ & $69(36 \%)$ & $192(100 \%)$ \\
\hline
\end{tabular}

\section{Methods}

Between April 1987 and February 1992, 209 consecutive patients attending the Diagnostic Clinic at Moorfields Eye Hospital with chlamydial oculogenital infection were retrospectively analysed. Cultures for Chlamydia trachomatis were performed from the conjunctivae of patients with conjunctivitis. Cultures were also performed from the cervix, urethra and rectum in women and urethra of heterosexual men who had or whose partners had chlamydial conjunctivitis. Screening for other genital infections was also performed. All men were screened for non-gonococcal urethritis $(\geqslant 10$ pus cells/HPF $\times 1000$ magnification in the urethral smear). According to the clinical findings and chlamydial culture results, the patients were categorised as above.

Serology was performed in all patients for syphilis and chlamydial IgG and IgM antibodies. A modified microimmunofluorescence test using pooled antigens was used to detect chlamydial antibodies. ${ }^{4}$

\section{Results}

Of all patients with proven chlamydial oculogenital infection $10.5 \%(22 / 209)$ and $94 \%$ $(197 / 209)$ had positive $\operatorname{IgM}(\geqslant 1: 8)$ and IgG $(\geqslant 1: 16)$ antibody titres to the $\mathrm{D}-\mathrm{K}$ serovars respectively, at initial examination (table 1). However, one IgG negative patient had a positive IgM chlamydial antibody titre and seven patients IgG negative at outset subsequently seroconverted on serial testing, leaving only four $(2 \%)$ patients with a persistent negative IgG response to chlamydia.

The geometric mean antibody IgG titres (GMT) were 1:98, 1:123, 1:245 and 1:101 in groups I to IV respectively. The IgG GMT values in control groups 1 and 2 were 1:45 and 1:36 respectively. Two of $86(2 \%)$ patients in group V (control group 1) who were of a similar age range had IgG antibody titres of $1: 32$ and $1: 64$. Only $1 / 55(1.8 \%)$ and $4 / 55(7 \cdot 3 \%)$ of patients in group VI (control group 2) had chlamydial IgG antibody titres of $\geqslant 1: 256$ and $\geqslant 1: 128$ respectively. No IgM response was seen in the control groups. In 192 patients who had serial serum specimens taken, IgG chlamydial antibody titres in blood rose by 4 -fold or more in $38(20 \%)$ patients within the first six weeks following presentation and in an additional $69(36 \%)$ patients titres fell by 4 -fold or more, during the same period. Thus in 107 out of 192 (56\%) patients a classical serodiagnostic response was shown (table 2).

Some low-titred cross-reactive IgG antibody response was seen in the majority of patients. Cross-reactivity between chlamydial species is shown in the Venn diagram (fig).

In the majority, $(802 / 836(96 \%))$ of the serological tests that showed cross-reactivity, the IgG antibody titres to other $C$ trachomatis serovars or chlamydial species were either equal $(224 / 836)$ [26.8\%] or lower or negative $(578 / 836[69 \%]$ than were the antibody titres to the $\mathrm{D}-\mathrm{K}$ serovars (table 3 ). 


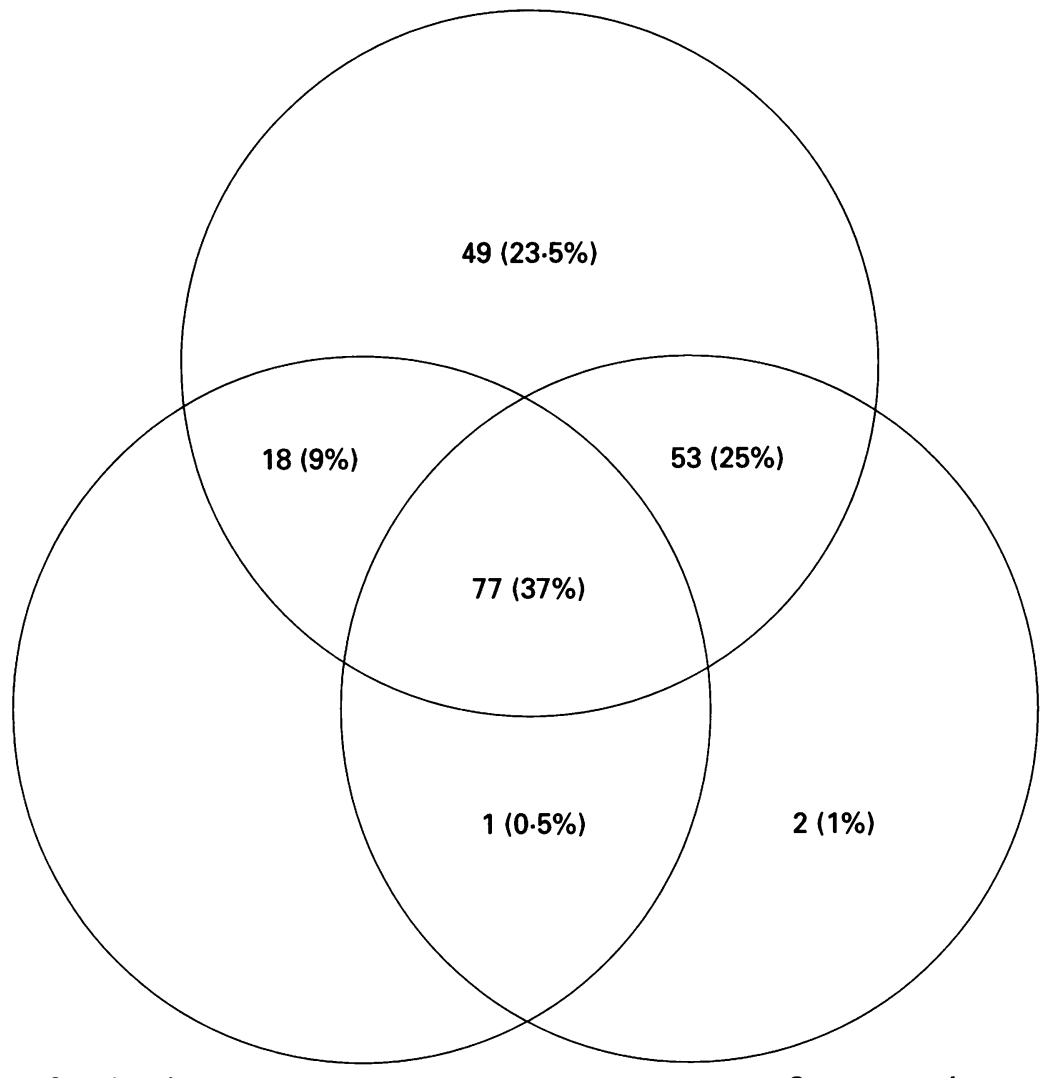

C. psittaci

C. pneumoniae

9 (4\%) Negative

IgG antibody cross reactivity to other species of chlamydia in patients with culture provcen C trachomatis.

\begin{abstract}
Discussion
Confirmation by culture or antigen detection with enzyme immunoassay (EIA), or direct immunofluorescence (DIF) are the preferred methods for diagnosing $C$ trachomatis infections. However, in many cases this is not possible because certain sites such as Fallopian tubes or lungs are difficult to sample and some patients have had prior anti-chlamydial antibiotics. In these cases, serology may be useful.

In this study the sensitivity, specificity, positive predictive value and negative predictive value for $C$ trachomatis infection at any site was $42 \cdot 6 \%, 98 \cdot 2 \%, 98 \cdot 8 \%$ and $31 \%$ respectively if the IgG chlamydial antibody titre was $\geqslant 1: 256$, when comparing groups I-IV with group VI as the negative control. Thus an IgG
\end{abstract}

Table 3 Cross-reactive IgG antibody titres in $C$ trachomatis oculogenital infection

\begin{tabular}{lcccc}
\hline Titre $^{*}$ & $C$ pneumoniae & $C_{\text {psittaci }}$ & $C$ trachomatis $(A-C)$ & $C$ trachomatis (L 1-3) \\
\hline Higher & $15(7 \%)$ & $2(1 \%)$ & $7(3 \%)$ & $10(5 \%)$ \\
Same & $17(8 \%)$ & $8(4 \%)$ & $86(41 \%)$ & $113(54 \%)$ \\
Lower & $102(49 \%)$ & $89(42 \%)$ & $98(47 \%)$ & $68(32 \%)$ \\
Negative & $75(36 \%)$ & $110(53 \%)$ & $18(9 \%)$ & $18(9 \%)$ \\
Total & $209(100 \%)$ & $209(100 \%)$ & $209(100 \%)$ & $209(100 \%)$ \\
\hline
\end{tabular}

${ }^{\star}$ titre compared with $C$ trachomatis (D-K) titre response of $\geqslant 1: 256$ is indicative of chlamydial infection in this group of patients.

IgM chlamydial antibody response was seen in the first serum specimens of only $10.5 \%(22 / 209)$ of patients with culture proven chlamydial oculogenital infection, whereas $25 \%$ of patients with ocular infection alone, showed an IgM response compared with $7-13 \%$ in those with genital infection with or without ocular involvement. The lower response in the latter groups may be due to asymptomatic or minimally symptomatic chlamydial genital infection that was present in most of our patients for some time before presenting with ocular infection. The transient IgM response seen in early chlamydial genital infection has often disappeared by this time. ${ }^{5}$

A positive chlamydial IgG antibody titre of $\geqslant 1: 16$ and $\geqslant 1: 64$ was seen in the first serum of $94 \%(197 / 209)$ and $77 \%(161 / 209)$ of all our patients with culture proven chlamydial infection respectively. Similar antibody responses are seen in patients with chlamydial ocular infection ${ }^{6}$ and pelvic inflammatory disease. $^{7}$ Treharne et al observed that $23 \%$ (83/143) and $62 \%(33 / 142)$ of women with acute salpingitis had a positive $\operatorname{IgM}(\geqslant 1: 8)$ and $\operatorname{IgG}(\geqslant 1: 64)$ response respectively. ${ }^{7}$

In this study $98.5 \%(128 / 130)$ of patients who had a chlamydia-positive ocular infection, with or without genital infection, had a positive serum IgG titre $(\geqslant 1: 16)$ to the $\mathrm{D}-\mathrm{K}$ serovars at initial examination compared with $2 / 86(2 \%)$ patients in the adenovirus conjunctivitis group. A positive chlamydial antibody IgG response in serum, in a patient with conjunctivitis in this study had a sensitivity, specificity, positive predictive value and negative predictive value of $98 \cdot 5 \%, 97 \cdot 7 \%, 98.5 \%$ and $97.7 \%$ respectively for chlamydial ocular infection. Darougar et al showed that the presence of antichlamydial IgG at a titre of $\geqslant 1: 32$ or IgM at a titre of $\geqslant 1: 8$ was closely associated with ocular paratrachoma while only $3 \%$ of 207 patients with non-chlamydial conjunctivitis had chlamydial antibodies in the serum. ${ }^{6}$

In individual patients the presence of high serum IgG titres $(\geqslant 1: 256)$, a four-fold or greater change in IgG titre, or the presence of serum IgM antibody may indicate chlamydial infection. In the highly selected group with conjunctivitis, chlamydial IgG antibody is a good indicator of chlamydial infection even at a low titre of $\geqslant 1: 16$. Serology therefore could be particularly useful in complicated cases such as children with chlamydial pneumonitis, or in patients partially treated with antibiotics.

Two percent (4/192) of patients were consistently negative for chlamydial IgG antibody on serial testing, although all these patients were found to have chlamydial genital infection by isolation, three were male and one was female. This level of serological non-reactivity is lower than other studies which showed that $10-20 \%$ of men with chlamydial urethral infection develop no detectable specific antibodies at all. ${ }^{89}$ In men, the absence of the 
antibody response may be because of weak antigenic stimulus at a very localised mucosal site.

The GMT of the antibody response in chlamydial oculogenital infection was lowest in those with primary genital infection, particularly in men, whilst it was highest in females who had dual ocular and genital chlamydial infection. This may be because of a greater antigenic stimulus and anamnestic response resulting from a second infected site, superimposed on long standing asymptomatic genital infection leading to a larger surface area of epithelium being involved. ${ }^{10}$ Cross reactive IgG antibody responses were seen between most chlamydial species and between the serovars of $C$ trachomatis. The IgG titre was the same $(26.8 \%)$, or lower or negative for chlamydial antibodies $(69 \%)$ than to that of the D-K serovars in patients with chlamydiapositive oculogenital infection, probably indicating serum cross-reactivity rather than an active infection due to other serotypes in these patients. Kuritsky et al showed that of 55 patients who had $\geqslant 4$ fold rise in titre to other respiratory pathogens, $3(5 \%)$ also had $\geqslant 4$ fold rise in IgM or IgG antibodies to $C$ trachomatis and seven of eight patients with psittacosis were also able to seroconvert to $C$ trachomatis serovars. ${ }^{11}$ Our study showed cross-reactive IgG antibody to the D-K serovars in $96 \%(802 / 836)$ of sera tested.

The type-specific micro-IF test, and to a greater degree genus-specific tests such as CFT, single-antigen immunofluorescence or elementary-body based ELISA antibody tests for the detection of chlamydial infection, may also demonstrate cross-reaction to $C$ pneumoniae. ${ }^{1213}$ This organism is now a recognised cause of respiratory infection and may be associated with myocarditis, chronic coronary heart disease, acute myocardial infarction and sarcoidosis. ${ }^{1415}$ Based on serological tests alone, $C$ trachomatis has been reported to cause community acquired pneumonia ${ }^{16}$ and myocarditis ${ }^{17}$ in adults. Because of this, many of the previously reported genus-specific serological studies or those which excluded this chlamydial species need to be reassessed using specific $C$ pneumoniae antigens. Although serovar and species-specific micro-IF tests are sensitive and can differentiate between species, they are technically difficult and only available in specialist laboratories. Cross reactions between chlamydial species and serovars even in micro-IF tests may give confusing results. Hence, it is desirable that cultures or chlamydial antigen detection methods such as EIA or DIF are performed to detect chlamydial infection and to assess the diagnostic significance of serologic tests. Antigenic cross-reactivity, polyclonal antibody stimulation, concomitant genital infection, or coexisting chlamydial respiratory pathogens may be important confounding and confusing factors in interpreting serologic tests for the diagnosis of chlamydial oculogenital infections. ${ }^{11}$ In the event that it is impractical to perform chlamydial culture or to carry out chlamydial antigen detection tests in support of clinical diagnosis, then the micro-IF serological test may be useful but should be interpreted with caution.

1 Stamm WE, Holmes KK. Chlamydia trachomatis infections of the adult. In: Holmes KK, Mardh PA, Sparling PF, Wiesner PJ, et al, 2nd ed. Sexually Transmitted Diseases 1990:181-93.

2 Treharne JD, Ballard RC. The expanding spectrum of chlamydia-a microbiological and clinical appraisal. In: Reviews in Medical Microbiology 1990;1:10-8.

3 Wang SP, Grayston JT, Alexander ER, Holmes KK Simplified microimmunofluorescence test with trachoma-lymphogranuloma venereum (Chlamydia trachoma-lymphogranulor chomatis) antigens for use as a scre.

4 Treharne JD, Darougar S, Jones BR. Modification of the microimmunofluorescence test to provide a routine serodiagnostic test for chlamydial infection. $f$ Clin Pathol 1977;30:510-7

5 Treharne JD. Chlamydial serodiagnosis-luxury or necessity? In: Proceedings of the European Society for Chlamydia Research. Bologna: Esculapio, 1988:230-3.

6 Darougar S, Treharne JD, Minassian D, El-Sheikh $H$ Dines RJ, Jones BR. Rapid serological test for diagnosis of chlamydial ocular infections. $\mathrm{Br} \mathcal{F}$ Ophthalmol 1978;62:503-8.

7 Treharne JD, Ripa RT, Mardh P-A, Svensson L, Westrom L, Darougar S. Antibodies to Chlamydia trachomatis in acute salpingitis. Br f Venereal Dis 1979;55:26-9.

8 Holmes KK, Hansfield HH, Wang SP, et al. Etiology of nongonococcal urethritis. $N$ Engl $f$ Med 1975;292: nongonococca

9 Taylor-Robinson D, Thomas BJ. The role of Chlamydia trachomatis in genital-tract and associated diseases. $\mathcal{F}$ Clin Pathol 1980;33:205-33.

10 Treharne JD, Forsey T, Thomas BJ. Chlamydial serology Br Med Bull 1983;39:194-9.

11 Kuritsky JN, Schachter J, Broome CV, Wilkinson HW, Wulff $\mathrm{H}$. Assessment of the microimmunofluorescence test for antibodies to Chlamydia trachomatis in adults with respiratory disorders. F Infect Dis 1983;148:1167-8.

12 Grayston JT, Kuo C, Wang SP, Altman J. A new Chlamydia psittaci strain, TWAR, isolated in acute respiChlamydia psittaci strain, TWAR, isolated in acute resp

13 Schachter J. Chlamydia psittaci-"re-emergence" of a forgotten pathogen. N Engl $\mathcal{F}$ Med 1986;315:189-91.

14 Marrie TJ, Grayston JT, Wang S-P, Kuo C-C. Pneumonia associated with the TWAR strain of Chlamydia. Ann Intern Med 1987;106:507-11.

15 Saikku P, Leininen M, Mattila $K$, et al. Serological evidence of an association of a novel chlamydia, TWAR, with chronic coronary heart disease and acute myocardial infarction. Lancet 1988;ii:983-6.

16 Komaroff AL, Aronson MD, Schachter J. Chlamydia trachomatis infection in adults with community-acquired pneumonia. $¥ A M A$ 1981;245:1319-22.

17 Odeh $M$, Oliven A, Rauchfleisch $S$, Eassan $H$. Dilated cardiomyopathy associated with Chlamydia trachomatis infection. F Intern Med 1991;229:289-91. 